\title{
Some Remarks on the Evaluation of Concentration Spectra in Solids From X-Ray Diffraction Profiles
}

\author{
P. Schattschneider and A. Wagendristel \\ Institut für Angewandte Physik der Technischen Hochschule Wien \\ (Z. Naturforsch. 30 a, 1261-1264 [1975] ; received July 11, 1975)
}

\begin{abstract}
The validity of some assumptions included in the theoretical basis of several $\mathrm{x}$-ray methods for the determination of concentration spectra in inhomogeneous solids, is analysed critically by theoretical considerations.
\end{abstract}

In order to observe homogenisation or compound formation in multicomponent powder compacts or layered structures x-ray diffraction methods can be successfully applied. The main merits of such techniques are non-destructivity and the possibility to obtain information about the concentration spectrum as well as about structural properties in the sample. Beside comparative methods making use of computer-synthesised diffraction profiles fitted to measured data ${ }^{1}$, several analytical techniques can be employed $^{2-5}$. The problem of the latter methods is to eliminate the instrumental function from the measured interference profile and furthermore to separate the physical influences, such as particle size broadening, the effect of internal strain and the spectrum of the lattice parameter.

It is the aim of the present work to compare previous approximate formulae relating the concentration spectrum to measured $x$-ray interference profiles with a more exact solution of this problem deduced in the following section.

\section{Theoretical Considerations}

The calculation is confined to a hypothetical sample consisting of randomly oriented crystallites showing uniform structure but different lattice parameters. The size of these crystallites is assumed to be sufficiently large thus allowing the neglect of particle size broadening. In addition the atoms shall be placed on the ideal sites corresponding to the particular concentration $c$, which is considered to be constant within each crystal.

With regard to the lattice of the component $\mathrm{A}$ represented by a triplet of lattice parameters $\left\{\bar{a}_{j}\right\}$, all alloy crystals are characterized by lattice dimensions $a_{j}(c)=\bar{a}_{j} \cdot\left[1+\Delta_{j}(c)\right]$. The direction of the incident and the reflected beam $\boldsymbol{s}_{0}$ and $\boldsymbol{s}$ resp., inclosing a fixed angle $2 \vartheta$ is expressed in coordinates of the reciprocal base $\left\{\boldsymbol{b}_{j}\right\}$ of the component $A$ by $\Delta \boldsymbol{s}=\lambda \sum_{j=1}^{3} \boldsymbol{b}_{j} h_{j} ;$ considering a volume element $\mathrm{d} h_{1} \mathrm{~d} h_{2} \mathrm{~d} h_{3}$ around $\Delta s$, the energy of the beam diffracted by a small crystal of concentration $c$ is given by ${ }^{6}$

$$
\begin{gathered}
P(c)=I_{0} N_{1} N_{2} N_{3} \cdot \frac{e^{4}}{m_{\mathrm{e}}{ }^{2} c^{4}} \cdot \frac{\lambda^{3} F^{2}(c, \vartheta) \cdot A(c, \vartheta) P(\vartheta)}{v \cdot \sin 2 \vartheta} \\
\cdot \tilde{I}\left(h_{1}, h_{2}, h_{3}, c\right) \cdot \mathrm{d} h_{1} \cdot \mathrm{d} h_{2} \cdot \mathrm{d} h_{3}= \\
=\prod_{j=1}^{3} N_{j} Q \frac{K(c, \vartheta)}{\sin 2 \vartheta} \\
\cdot I\left(h_{1}, h_{2}, h_{3}, c\right) \mathrm{d} h_{1} \cdot \mathrm{d} h_{2} \cdot \mathrm{d} h_{3}
\end{gathered}
$$

with

$$
\begin{array}{r}
\tilde{I}\left(h_{1}, h_{2}, h_{3}, c\right)=\prod_{j=1}^{3} \sum_{l_{j}=-N_{j}}^{N_{j}}\left(1-\left|l_{j}\right| / N_{3}\right) \\
\cdot \exp \left\{2 \pi i \cdot h_{j} l_{j} \cdot\left[1+\Delta_{j}(c)\right]\right\} .
\end{array}
$$

$I_{0} \quad$ intensity of incident radiation,

$N_{j} \quad$ number of elementary cells in direction $j$ in the crystal,

$F^{2}(c, \vartheta) \quad$ scattering factor of the unit cell,

$v \quad$ volume of the unit cell in the reference lattice (component $A$ ),

$e^{4} / m_{\mathrm{e}}{ }^{2} c^{4} \quad$ scattering factor of a single electron,

$P(\vartheta)=1 / 2\left(1+\cos ^{2} \vartheta\right) \quad$ Polarisation factor,

$l_{j} \quad$ spacing between elementary cells along

direction $j$ in units $a(c)$,

$A(c, \vartheta) \quad$ absorption factor of the radiation in the

$\lambda \quad$ sample,

$Q \quad$ factor, including all coefficients independent of $c$ and $\vartheta$.

Considering $M$ randomly oriented crystals, then, in order to account for the contribution of each crystal to the diffracted energy, the element $\mathrm{d} h_{1} \mathrm{~d} h_{2} \mathrm{~d} h_{3}$ has to scan along a sphere $|\Delta \boldsymbol{s}|=$ constant, i.e. $h_{1}, h_{2}$, and $h_{3}$ have to be varied, so that $2 \vartheta$ remains fixed. We may assume that $P(c)$ has a significant value only between the reciprocal lattice points $\left(\bar{h}_{1} \bar{h}_{2} \bar{h}_{3}\right)$ of component $A$ and $B$ and 
a small region around them. In the cubic system it is possible to find an orthorhombic base so that $\left(\bar{h}_{1} \bar{h}_{2} \bar{h}_{3}\right)$ is transformed to $\left(00 \bar{h}_{3}\right)$. Then, in a small region around $\left(00 \bar{h}_{3}\right)$ the directions $j=1$ and $j=2$ are tangential to the sphere $|\Delta s|=$ const, whereas $\lambda h_{3}=|\Delta s|=2 \bar{a}_{3} \sin \vartheta$. Hence we may write for the energy obtained in an interval $\mathrm{d} 2 \vartheta$ around the angle $2 \vartheta$ with respect to the direction of incidence

$$
\begin{aligned}
& \bar{I}(c, 2 \vartheta)=M \cdot N_{1} \cdot N_{2} \cdot N_{3} \cdot m_{\left(00 \overline{h_{3}}\right)} \cdot \\
& \quad \frac{b \cdot Q \cdot K(c, \vartheta)}{4 \pi \cdot \sin ^{2} \vartheta \cdot \cos \vartheta} \cdot \frac{\mathrm{d} h_{3}}{\mathrm{~d} 2 \vartheta} \cdot \int_{-1 / 2}^{1 / 2} \tilde{I} \cdot \mathrm{d} h_{1} \cdot \mathrm{d} h_{2}
\end{aligned}
$$

since only a fraction $m_{\left(00 \overline{h_{s}}\right)} M b \cos \vartheta / 2 \pi \sin 2 \vartheta$ will be registered at the counter showing a slit of breath $b$. In order to account for the contribution of all equivalent regions along the sphere $|\Delta \boldsymbol{s}|=$ const the recurrence factor $m$ had to be introduced. The interval of integration seems to be chosen without any motive, but, as we shall see later it is only essential to consider limits of integration at which the integrant is already negligibly small. For large numbers $N_{1}, N_{2}$ the limits could be taken much more narrow, thus justifying the approximation of the sphere by the tangential plane. If particle size broadening should be sufficiently small we have to consider the case $N_{j} \rightarrow \infty$. The expression

$$
\begin{aligned}
I=\int_{-1 / 2}^{1 / 2} \sum_{-N}^{N}(1-|l| / N) \cdot \\
\cdot \exp \{2 \pi i \cdot h \cdot l \cdot[1+\Delta(c)]\} \mathrm{d} h
\end{aligned}
$$

is split as follows

$$
\begin{aligned}
I= & \int_{-1 / 2}^{1 / 2} \lim _{N \rightarrow \infty} \sum_{-N}^{N} \cdot \exp \{2 \pi i \cdot h \cdot l \cdot[1+\Delta(c)]\}- \\
& -\lim _{N \rightarrow \infty} \frac{1}{N} \sum_{-N}^{N}|l| \cdot \frac{\sin \pi \cdot l \cdot[1+\Delta(c)]}{\pi \cdot l \cdot[1+\Delta(c)]} .
\end{aligned}
$$

Recognizing that the periodic Dirac $\delta$-function is given by

$$
\lim _{N \rightarrow \infty} \sum_{-N}^{N} \exp (2 \pi i \cdot l \cdot x)=\sum_{Z=-\infty}^{\infty} \delta(x-Z)
$$

where $Z$ is an integer, the first term gives $[1+\Delta(c)]^{-1}$ whereas the second tends to zero as can be easily shown. We obtain

$$
\lim _{N \rightarrow \infty} I_{j}=1 /\left[1+\Delta_{j}(c)\right] \text {. }
$$

This is consistent with the requirement of the diffracted energy being independent of the chosen reciprocal base. [In its own base a crystal of concentra- tion $c$ would show an energy according to Eq. (1) with $\Delta=0$ and $v=v(c)=\prod_{j=1}^{8}\left(1+\Delta_{j}\right) v_{A}$ ]. We finally obtain an energy diffracted by one unit cell of

$$
\begin{aligned}
\bar{I}(c, 2 \vartheta)= & \frac{I(c, 2 \delta)}{M \cdot N_{1} \cdot N_{2} \cdot N_{3}}=\frac{Q^{\prime} \cdot K(c, \vartheta)}{\sin ^{2} \vartheta} . \\
& \cdot \prod_{j=1}^{2} \frac{1}{1+\Delta_{j}(c)} \sum_{l=-N_{3}}^{N_{3}}\left(1-|l| / N_{3}\right) \\
& \cdot \exp \left\{2 \pi i \cdot h_{3}(\vartheta) \cdot l \cdot\left[1+\Delta_{3}(c)\right]\right\}
\end{aligned}
$$

for crystals of the concentration $c$. Considering a sample showing a spectrum of lattice parameters expressed by an abundance distribution of elementary cells

$$
\frac{\mathrm{d} n(c)}{\mathrm{d} c}= \begin{cases}H(c) & {[0,1]} \\ 0 & \text { elsewhere },\end{cases}
$$

we may write for the intensity at a fixed diffraction angle

$$
\begin{gathered}
I(2 \vartheta)=\int_{0}^{1} \bar{I}(c, 2 \vartheta) \mathrm{d} c=\frac{Q^{\prime}}{\sin ^{2} \vartheta} \cdot \int_{0}^{1} H^{\prime}\left(c^{\prime}, \vartheta\right) \\
\cdot \sum_{-N_{3}}^{N_{3}}\left(1-|l| / N_{3}\right) \cdot \exp \left\{2 \pi i \cdot h_{3}(\vartheta) \cdot l \cdot\right. \\
\left.\cdot\left[1+\Delta_{3}(c)\right]\right\} \mathrm{d} c
\end{gathered}
$$

with

$$
\begin{aligned}
H^{\prime}(c, \vartheta)=H(c) \cdot K(\vartheta, c) \cdot\left[1+\Delta_{1}(c)\right]^{-1} \\
\cdot\left[1+\Delta_{2}(c)\right]^{-1} .
\end{aligned}
$$

So far, limited lattice coherency along $h_{3}$ is still included $\left(N_{3} \neq \infty\right)^{*}$.

The following definitions are introduced: $h_{3}=h$, $\Delta_{3}=\Delta, \quad z=h[1+\Delta(c)]=z(h, c)$. Together with $c=c(h, z)$ giving $\mathrm{d} c=\mathrm{d} z(h \mathrm{~d} \Delta / \mathrm{d} c)^{-1}$, the following expression is obtained

$$
\begin{array}{r}
I(2 \vartheta)=\frac{Q^{\prime}}{\sin ^{2} \vartheta} \int_{h}^{h 1+\Delta(1)]} H^{\prime \prime}[z, h(\vartheta)] \cdot \sum_{-N_{3}}^{N_{3}}\left(1-|l| / N_{3}\right) \\
\exp \{2 \pi i \cdot l \cdot z\} \cdot \mathrm{d} z
\end{array}
$$

with

$$
H^{\prime \prime}(z, h)=H^{\prime}[c(h, z)] / h(\mathrm{~d} \Delta / \mathrm{d} c) .
$$

The integral Eq. (12) relates the diffraction profile to the concentration spectrum $H(c)$. It has to be

* To account for it, the function $\exp 2 \pi i h_{3} \Delta_{3}(c)$ is as sumed to vary slowly with $h_{3}$ because of the small value for $\Delta_{3}$, which enables the approximation $h_{3} \approx h_{3}$. Then, the intensity can be expressed as a Fourier series in $h_{3} 4,5$. 
solved for $H^{\prime \prime}(z, h)$. Using again $(6)^{* *}$ we obtain similarly as in the case $N_{1}, N_{2} \rightarrow \infty$

$$
I(2 \vartheta)=\left(Q^{\prime} / \sin ^{2} \vartheta\right) \cdot H^{\prime \prime}\left[z=\bar{h}_{3}, h(\vartheta)\right]
$$

for $N_{3} \rightarrow \infty$, since the only singularity within the interval of integration exists at $z=\bar{h}_{3}$. Substituting back $z=\bar{h}_{3}=h(\vartheta)[1+\Delta(c)]$ and $h(\vartheta) \lambda=2 \bar{a}_{3} \sin \vartheta$ we obtain in terms of $c$

with

$$
H(c)=F \cdot I[2 \vartheta(c)]
$$

$F=\sin ^{2} \vartheta(c) \cdot\left[1+\Delta_{1}(c)\right] \cdot\left[1+\Delta_{\mathbf{2}}(c)\right] \cdot$

$$
\cdot h[\vartheta(c)] \cdot \frac{\mathrm{d} \Delta_{3}}{\mathrm{~d} c} / Q^{\prime} \cdot K[c, \vartheta(c)]
$$

and

$$
\vartheta=\arcsin \left\{\left(\lambda \bar{h}_{3}\right) /\left(\bar{a}_{3}\left[1+\Delta_{3}(c)\right]\right) .\right.
$$

\section{Discussion}

The usual way to calculate diffraction profiles of inhomogeneous crystalline solids is to assume large crystallites a priori. The contribution of crystals showing a concentration around $c$ is evaluated in their inherent reciprocal base leading to

$$
\mathrm{d} I[2 \vartheta(c)]=\frac{Q^{\prime} \cdot K(c, \vartheta) \cdot H(c) \mathrm{d} c}{v(c) \cdot \sin ^{2} \vartheta}
$$

which gives the same result as that obtained in this work. In the paper ${ }^{3}$, however, the factor $v(c)$ is neglected which causes an inexact expression $H(c)_{3}$ instead of $H(c)$. From this concentration spectrum the penetration curve is deduced using again an incorrect formula $x(c)_{3} \propto \int_{0}^{c} a\left(c^{\prime}\right) H\left(c^{\prime}\right)_{3} \mathrm{~d} c^{\prime}$. But since this expression is approximated by $\langle a\rangle \int_{0}^{c} H\left(c^{\prime}\right)_{3} \mathrm{~d} c^{\prime}$, the previous error is compensated. As shown in ${ }^{5}$ the correct formula would be $x(c) \alpha \int_{0}^{c} v\left(c^{\prime}\right) H\left(c^{\prime}\right) \mathrm{d} c^{\prime}$ which is essentially the same as $\int_{0}^{c} H\left(c^{\prime}\right)_{3} \mathrm{~d} c^{\prime}$. In the papers ${ }^{4,5}$ the contribution of particle size broadening is also considered. Contrary to large crystals giving sharp reflections only when the Bragg equation is satisfied, small particles are still able to scatter when they are somewhat twisted out of the Bragg position. Hence the treatment had to be carried out in the same way as shown in the previous section, i.e. at a fixed diffraction angle the contributions of

** At this point it should be mentioned that the $\delta$-function should be applied with great care. In the present case, however, a strict derivation leads to the same results ${ }^{8}$. all concentrations and all crystal orientations have to be summed up. The finite sums appearing in Eq. (4) have been assumed to differ not very much from 1 . Warren ${ }^{7}$ showed that only the coherence length along $j=3$ is responsible for the particle size broadening of the diffraction profile. Therefore it seems better to replace the finite sums by their limiting values for $N_{1}, N_{2} \rightarrow \infty$ as done in Equation (7). This would satisfy the condition that after the elimination of the particle size broadening the reflection has to be described by Equation (8).

As already mentioned the step necessary to separate the influence of small coherence length from the concentration spectrum is to approximate the variable $\bar{h}_{3}$ by the Miller index $\bar{h}_{3}$ in $\exp \left\{2 \pi i h_{3} \Delta_{3}(c)\right\}$. This has been established by the validity of Vegard's law $\Delta_{3}(c)=k c$ with $k$ very small. In this case the diffraction profile can be expressed as a Fourier series with coefficients being products of the known particle size coefficient $A_{n}=\left(1-n / N_{3}\right)$ and $A_{n}{ }^{c}$ as well as $B_{n}{ }^{c}$, which are related to the concentration spectrum by

$$
\begin{aligned}
H^{\prime}(-c)=\sum_{0}^{\infty} A_{n}{ }^{c} \cos 2 \pi \bar{h}_{3} k c \cdot n+ & \\
& +B_{n}{ }^{c} \sin 2 \pi \bar{h}_{3} k c \cdot n .
\end{aligned}
$$

The negative sign arises from the fact that $c$ is equal to $\left(\bar{h}_{3}-h\right) / k \bar{h}_{3}$, corresponding to the smaller diffraction angle of the component with the larger lattice parameter. Although this negative sign has not been accounted for in ${ }^{4,5}$ a correct relation was obtained, since it was suggested to perform the separation of the concentration spectrum from the diffraction profile directly in terms of $\vartheta$ instead of $h_{3} .2 \vartheta_{\mathrm{A}}$ corresponds to $c=0$, and $2 \vartheta_{\mathrm{B}}$ to $c=1$. The relation between $2 \vartheta$ and $c$, i.e. the scale in $c$ between $2 \vartheta_{\mathrm{A}}$ and $2 \vartheta_{\mathrm{B}}$, however, differs from the exact expression $\vartheta=\arcsin 2 \lambda h_{3} / \bar{a}_{3}(1+k c)$ by $\vartheta=\arcsin 2 \lambda \bar{h}_{3}(1-k c) / \bar{a}_{3}$ as a result of the Fourier development. But as the Fourier method is restricted to $k=\Delta a / \bar{a}_{3} \ll 1$ the difference is expected to be small because of $(1-k c) \approx(1+k c)^{-1}$.

So, in order to deduce the concentration spectrum from the diffraction profile after the elimination of all other contributions, we finally have to suggest the use of the Eq. (15), (16), (17) rather than the previously given formulae.

\section{Acknowledgements}

The authors are indepted to the Austrian Fond zur Förderung der wissenschaftlichen Forschung for the sponsorship of this work. 
1 J. Unnam, J. A. Carpenter, and C. R. Houska, J. Appl. Phys. 44, 1957 [1973].

2 Rev. of literature in: C. R. Houska, J. Appl. Phys. 41, 69 [1970].

3 P. S. Rudman, Acta Cryst. 13, 905 [1960].

4 A. Wagendristel and D. Wolf, Z. Metallkunde 65, 660 [1974].
5 A. Wagendristel, Appl. Phys. 5, 471 [1975].

6 A. H. Compton and S. K. Allyson, x-Rays in Theory and Experiment, D. Van Nostrand, New York 1963.

7 B. E. Warren and B. L. Averbach, J. Appl. Phys. 21, 595 [1950].

8 P. Schattschneider, Thesis, T.U. Vienna, in preparation. 